\title{
CHEMOKINE STORM
}

\section{Carlos Rodriguez \\ 1 Fundación Jiménez Díaz}

Potential competing interests: The author(s) declared that no potential competing interests exist.

A severe reaction in which the body releases too many chemokines into the blood or into tissues too quickly. Chemokines play a fundamental role as cell chemoattractants in normal and pathological immune responses as well as in tissue homeostasis and tissue repair, but having a large amount of them released in the body all at once can be harmful. A Chemokine Storm can occur as a result of an infection, autoimmune condition, Acute Respiratory Distress Syndrome (ARDS), sepsis, cancer or other diseases. It may also occur after treatment with some types of immunotherapy. Due to the massive mobilization and activation of inflammatory cells during a Chemokine Storm, signs and symptoms can include high fever, inflammation (redness and swelling), and severe fatigue and nausea. 